\title{
Channel Estimation with linear Interpolation and Decision Feedback for UTRA FDD Downlink
}

\author{
Klaus Knoche, Jürgen Rinas and Karl-Dirk Kammeyer \\ University of Bremen, FB-1, Department of Telecommunications \\ P.O. Box 3304 40, D-28334 Bremen, Germany, \\ Fax: +(49)-421/218-3341, e-mail: knoche@comm.uni-bremen.de
}

\begin{abstract}
This paper introduces a Channel Estimation (CE) scheme called Linear Interpolation with Decision Feedback (LIDF) for downlink coherent Rakecombining in a DS-CDMA mobile environment. In this case an UTRA FDD scenario is taken for showing the feasibility of this approach. This scheme uses known periodically time-multiplexed pilot symbols for interpolating the channel coefficients in between. It decides all symbols within the focused slot and takes them to do a new channel estimation using a linear regression to refine its estimation. Beside of using this LIDF on pilot sequences of every Dedicated Physical Channel (DPCH) in UMTS, the refinement of the estimation by regression could also be used for the Common Pilot Channel defined in the 3GPP UTRA FDD DL specification [1]. This main approach has been done for 2-G systems before i.e. [2] and is now implemented for 3GPP UTRA FDD DL.
\end{abstract}

The basic advantage of this approach is to have good noise reduction capability and good adaptation to the time variant channel coefficients. Some simulation results for one and multiple users for the LIDF-CE compared with other well known CE-filters are presented.

\section{INTRODUCTION}

UMTS is the 3rd generation mobile cellular communication system of the (near) future. Upon others it defines a FDDWide-Band-CDMA scheme using a coherent Rake-receiver as shown in fig. 1. The signal $\mathbf{y}_{I D}$ which can be detected after the I\&D-Operation is represented as ${ }^{1}$

$$
y_{I D, l}(i)=\frac{1}{S F} \sum_{j=i \cdot S F}^{(i+1) \cdot S F-1} x_{l}(j) \cdot \frac{1}{\sqrt{2}} \cdot c_{s c r}^{*}(j) \cdot c_{c h}(j)
$$

where $c_{c h}(j)$ is the real valued channelization code also known as OVSF-code with spreading factor $(S F)$ and $c_{s c r}$ represents the complex scrambling code.

The received signal after maximum ratio combining for a $L_{R}$-finger Rake-receiver is denoted as

$$
y_{M R C}(i)=\sum_{l=0}^{L_{R}} y_{I D, l}(i) \cdot \hat{h}_{l}^{*}(i)
$$

where $\hat{h}_{l}(i)$ is the estimated channel coefficient for the $l$ th finger. In UMTS-FDD downlink a Dedicated Physical

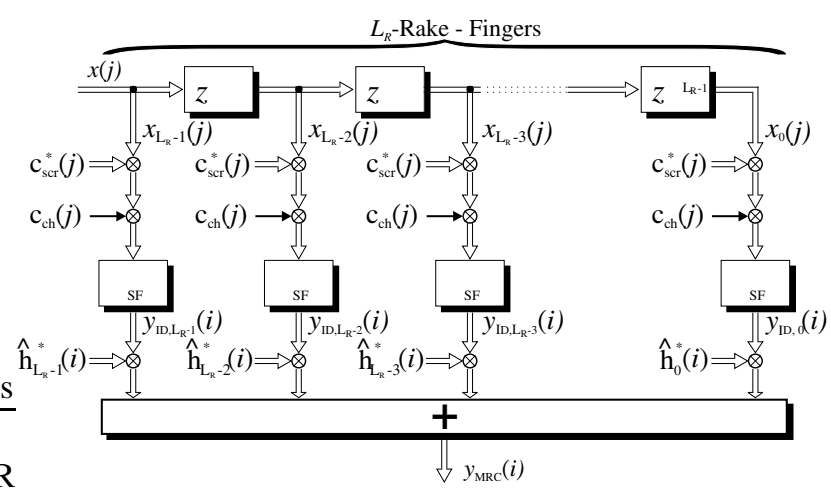

Figure 1: Rake-receiver

Channel (DPCH) consists of user data also called Dedicated Physical Data Channel (DPDCH), time-multiplexed with control information - the so called Dedicated Physical Control Channel (DPCCH) as depicted in fig. 2 or in [1]. The DPCCH itself is divided in Transmit Power Control (TPC) symbols, a Transport Format Combination Indicator (TFCI), and a pilot sequence. Fifteen slots with $M_{\text {slot }}=2560$ chips using this structure are combined in one frame of $10 \mathrm{~ms}$ length. The pilot sequence changing for every slot within a frame is used for channel estimation by correlating the incoming signal with the pilot sequence. Besides of this pilot symbol aided scheme, 3GPP also provides pilot channels for CE. This Common Pilot Channel is not considered within this paper, although this here proposed scheme can be easily adapted to it. Let

$$
N_{D}=N_{\text {slot }}-N_{\text {pilot }} \quad \text { with } \quad N_{\text {slot }}=M_{\text {slot }} / S F
$$

where $N_{\text {pilot }}$ denotes the number of pilot symbol used in DPCCH. We can estimate a channel coefficient in centre of the pilot sequence in the $\nu$-th slot by correlation.

$$
\hat{h}_{l, \nu}=\frac{1}{N_{\text {pilot }}} \sum_{k=N_{D}}^{N_{\text {slot }}-1} y_{I D, l}(k) \cdot \frac{1}{\sqrt{2}} \cdot p_{\nu}^{*}\left(k-N_{D}\right)
$$

with the pilot sequence $p_{\nu}(k)$ defined as:

$$
p_{\nu}(k)\left\{\begin{array}{lll}
\neq 0 & : & 0 \leq k<N_{\text {pilot }} \\
=0 & : & \text { otherwise. }
\end{array}\right.
$$

\footnotetext{
${ }^{1}(*)$ denotes conjugate complex
} 


\begin{tabular}{|c|c|c|c|c|}
\hline \multicolumn{3}{|c|}{ 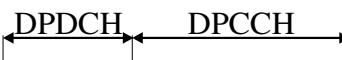 } & DPDCH & $\stackrel{\mathrm{DPCCH}}{\longrightarrow}$ \\
\hline Data1 & $\mathrm{TPC}$ & TFCI & Data2 & Pilot \\
\hline \multicolumn{5}{|c|}{$\mathrm{M}_{\mathrm{slot}}=2560$ chips $=10 * 2^{\mathrm{k}}$ bits $(\mathrm{k}=0 . .7)$} \\
\hline \multicolumn{5}{|c|}{ DPCH (1 Slot) } \\
\hline Slot 0 & Slot 1 & & \begin{tabular}{l|l} 
Slot i & \\
\end{tabular} & Slot14 \\
\hline \multicolumn{5}{|c|}{ frame, $T_{\mathrm{f}}=10 \mathrm{~ms}$} \\
\hline
\end{tabular}

Figure 2: Slot and frame structure

The estimates $\hat{h}_{l, \nu}$ are processed further on, using CEfilters. In general signal processing has to cope with noise and the time variant change of channel coefficients. Unfortunately both effects are contradictory to each other. In order to antagonise noise averaging is mandatory but this will deteriorate the performance in a high mobile environment. Therefore a compromise has to be found. There are two standard linear CE-filters discussed in [3] which will be used as reference in this paper.

The averaging filter also shown in fig. 3a) tries to reduce the noise and is not tracking the channel coefficient within a slot at all. The linear interpolation-filter is just drawing a line between two neighbouring estimates to follow the change of the time variant channel coefficient of one Rakefinger as shown in fig. $3 b$ ). Therefore noise reduction of the linear interpolation CE depends on the position within the slot. In linear interpolation, there is no noise reduction at the position of the pilot and up to three $\mathrm{dB}$ in the middle between two pilot sequences. In order to examine the per-
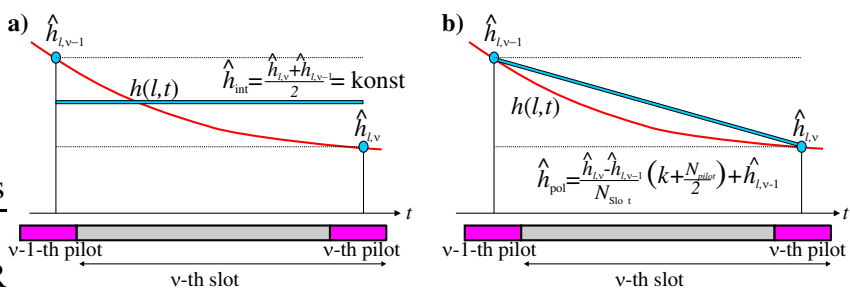

Figure 3: Classical CE, (a): Averaging, (b): Linear interpolation

formance of these two filters a bit further the Mean Squared Error (MSE) between the "real" channel coefficient $h_{\nu}(i)$ and its estimation $\hat{h}_{x x x, \nu}(i)$ with $x x x$ denotes the estimation scheme, can be expressed as:

$$
M S E_{\nu}(i):=\mathrm{E}\left\{\left|\hat{h}_{x x x, \nu}(i)-h_{\nu}(i)\right|^{2}\right\}
$$

In this case expectation is substituted by time average due to ergodicity

$$
\operatorname{MSE}(i)=\lim _{N \rightarrow \infty} \frac{1}{N} \sum_{\nu=0}^{N-1}\left|\hat{h}_{x x x, \nu}(i)-h_{\nu}(i)\right|^{2}
$$

with $i \in\{0, \ldots, 2560 / S F-1\}$. The estimate $\hat{h}_{x x x}$ is the estimate of the considered CE $\mathrm{xxx}$ and $h_{\nu}(i)=$
$\sum_{k=i}^{i+S F-1} h_{\nu}(k)$ where $h_{\nu}(k)$ is the real channel coefficient calculated for every chip .

Using a one tap Rayleigh channel at a velocity $v$ with an $E_{B} / N_{0}$ of $8 \mathrm{~dB}$ and the UMTS-transmission slot format \#10 ( $\mathrm{SF}=128,4$ pilot symbols per sequence) the MSE depends on the position within the slot. This is depicted for the two slot averaging CE in fig. 4. A global minimum

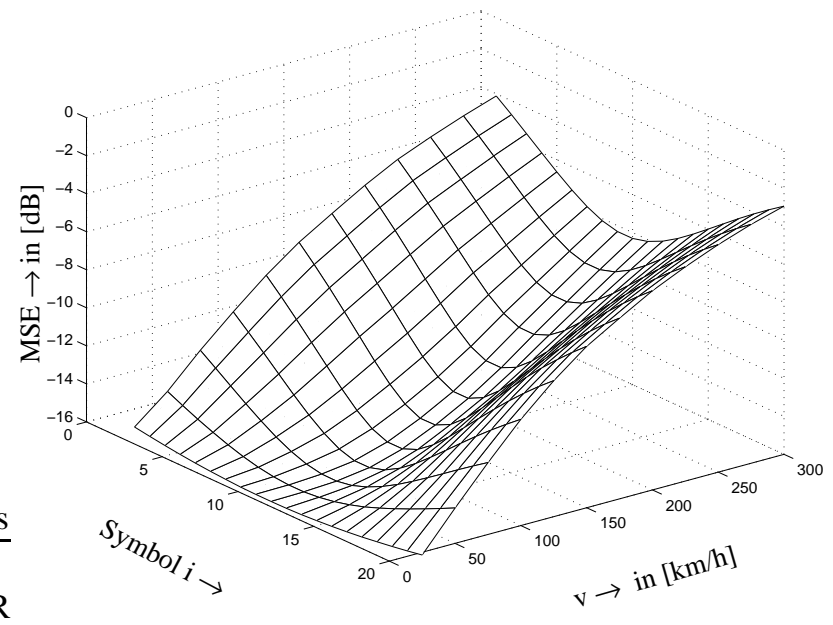

Figure 4: MSE of averaging CE

in the middle between two pilot sequences can be seen in fig. 4 according to the intersection of the estimated and the "real" channel coefficient in fig. 3a. Especially for higher velocities this method degrades fast, but for lower velocities it yields a $3 \mathrm{~dB}$ gain compared to a single slot $\mathrm{CE}$. In fig. 5 the MSE for the linear interpolation is depicted. At

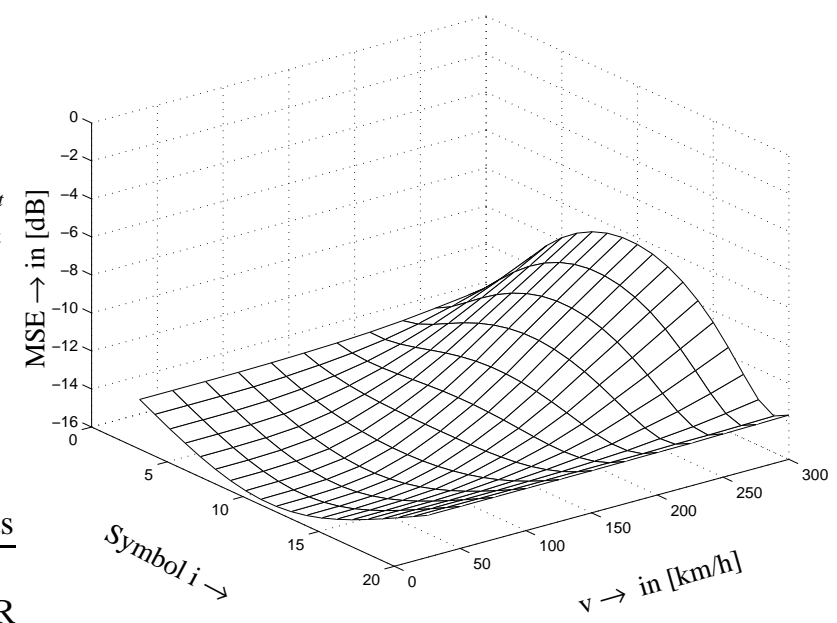

Figure 5: MSE of linear interpolation CE

the edges (near the pilot sequences) and for low velocities this method provides a three $\mathrm{dB}$ loss against the averaging $\mathrm{CE}$, as expected, it reaches the same MSE as the averaging $\mathrm{CE}$ in the middle between two pilot sequences. On the other hand it provides a much better behaviour in a highly mobile environment. For higher velocities the advantage of $3 \mathrm{~dB}$ noise reduction in the middle will be countered by ascending nonlinearity of the real channel coefficient resulting in a global maximum at the centre, that can also be deduced 
from fig. 3b).

\section{LINEAR INTERPOLATION WITH DECI- SION FEEDBACK}

The principle of the LIDF-scheme is depicted in fig. 6. First

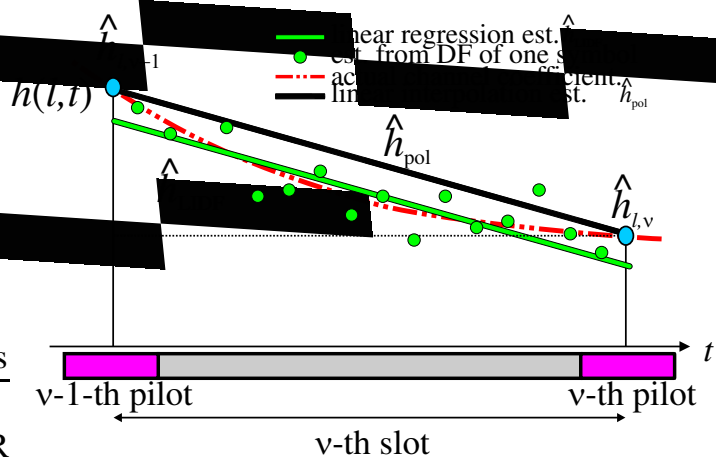

Figure 6: Principle of LIDF-CE

we use the linear interpolation scheme described in the previous section for an initial channel estimation.

$$
\begin{aligned}
\hat{h}_{\text {Pol }, \nu, l}(i)= & \frac{N_{\text {slot }}-\left(N_{\text {pilot }} / 2+i\right)}{N_{\text {slot }}} \cdot \hat{h}_{\nu-1, l} \\
& +\frac{N_{\text {pilot }} / 2+i}{N_{\text {slot }}} \cdot \hat{h}_{\nu, l} \\
\text { for } \quad 0 \leq i<N_{\text {slot }} & 0 \leq
\end{aligned}
$$

Those estimated channel coefficients $\hat{h}_{\mathrm{Pol}, \nu, l}(i)$ are used to decide the data. Note that in this paper the decision is made without utilising a FEC. A decision after channel decoding would be much more reliable and therefore improve the system significantly but computational costs and delay of the system will also raise. Esspecially in its practcal implementation, this would lead to save all data $\mathbf{y}_{I D}$ and wait until channel coding including interleaving is done. Therefore a vast amount of memory is needed, which is not possible for a handheld to bear due to its costs and power consumption.

$$
\begin{gathered}
\hat{d}_{\mathrm{Pol}, \nu}(i)=\frac{1}{\sqrt{2}} \operatorname{sign}\left(\sum_{l=0}^{L-1} y_{\mathrm{ID}, l}(i) \cdot \hat{h}_{\mathrm{Pol}, \nu, l}^{*}(i)\right) \\
\text { for } 0 \leq i<N_{D}
\end{gathered}
$$

Pilot symbols are not detected because they are known anyway.

$$
\begin{array}{r}
\hat{d}_{\mathrm{Pol}, \nu}(i)=\frac{1}{\sqrt{2}} p_{\nu}\left(i-N_{\mathrm{D}}\right) \\
\text { for } \quad N_{\mathrm{D}} \leq i<N_{\text {slot }}
\end{array}
$$

After deciding the data symbols using a regular Rakereceiver, this data can be used to estimate the channel coefficients $\hat{h}_{\mathrm{PolDF}, \nu, l}(i)$ for every symbol within the slot.

$$
\hat{h}_{\mathrm{PolDF}, \nu, l}(i)=y_{\mathrm{ID}, l}(i) \cdot \hat{d}_{\mathrm{Pol}, \nu}^{*}(i)
$$

Unfortunately there is a possibility of error in the decision making process, especially in this case without using any FEC. To overcome or at least alleviate this obstacle, some sort of averaging has to be done. In this case we propose a model based approach for the progression of the channel coefficients. A linear regression line $y=a x+b$ is employed. In general a linear regression with $n$ is the number of measured points (here $n=2560 / S F$ per slot) and $x_{i}$ is the position of the $i$-th measurement and $y_{i}$ is the appropriate measured value, is explained in [4, S.696ff.].

$$
\begin{gathered}
a=\frac{n \sum_{i=0}^{n-1} x_{i} y_{i}-\left(\sum_{i=0}^{n-1} x_{i}\right)\left(\sum_{i=0}^{n-1} y_{i}\right)}{n \sum_{i=0}^{n-1} x_{i}^{2}-\left(\sum_{i=0}^{n-1} x_{i}\right)^{2}} \\
b=\frac{1}{n} \sum_{i=0}^{n-1} y_{i}-a \frac{1}{n} \sum_{i=0}^{n-1} x_{i}
\end{gathered}
$$

To estimate $\hat{h}_{\mathrm{LIDF}, \nu, l}(i)$ equations 12 and 13 are used separately for real and imaginary part on $\hat{h}_{\mathrm{PolDF}, \nu, l}(i)$, yielding the regression coefficients $a_{\text {real }, \nu, l}, b_{r e a l, \nu, l}$, $a_{i m a g, \nu, l}$ and $b_{i m a g, \nu, l}$.

$$
\begin{aligned}
& \hat{h}_{\mathrm{LIDF}, \nu, l}(i)= a_{\text {real }, \nu, l} \cdot i+b_{\text {real }, \nu, l} \\
&+j\left(a_{\text {imag }, \nu, l} \cdot i+b_{\text {imag }, \nu, l}\right) \\
& \text { for } \quad 0 \leq i<N_{\text {slot }}
\end{aligned}
$$

Comparing the MSE depicted in fig. 7 for the decision feed-

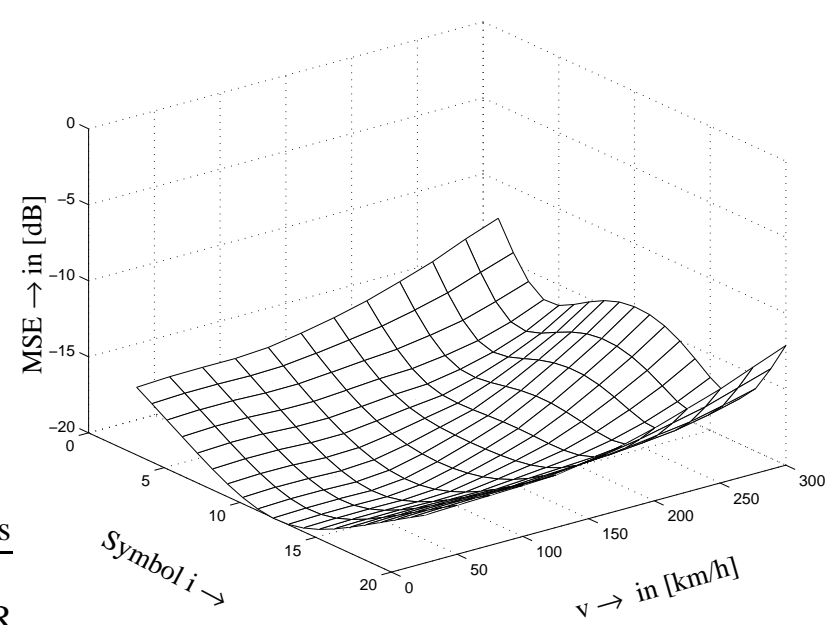

Figure 7: MSE of the Linear Interpolation with Decision Feedback scheme

back and fig. 5 for the linear interpolation scheme. Some differences can be worked out. For small velocities both look quite similar, but the decision feedback scheme has much better (smaller) MSE because of its better noise reduction capabilities. In a high mobile environment some deviations at the borders of the slot can be seen. These deviations are the result of the non linear characteristics of the channel coefficients. In this case a systematic error is induced, but in general this error is neglectable. This problem can also be seen in fig. 6 , where the line for the estimate 
crosses the curve for the true channel coefficients at two points which results in two minima within the MSE-plot in fig. 7. For some special high speed cases, it may be more advantageous to use a higher order regression type.

\section{Simulation Results}

In this section simulation results are presented. The slot formats taken for simulation are shown in table II. The complete list of slot formats can be found in [1]. A Vehicular-A channel with fixed delays for the channel-taps is taken, as described in [5].

\section{TABLE II}

Used slot formats within the simulations

\begin{tabular}{|lllcc|}
\hline $\begin{array}{l}\text { Slot } \\
\text { Format }\end{array}$ & $S F$ & \multicolumn{2}{c|}{$\begin{array}{c}\text { DPCH } \\
\text { in Symbols per }\end{array}$} & $\begin{array}{c}\text { DPDCt } \\
\text { in }\end{array}$ \\
\hline$\# 8$ & 128 & 20 & 17 & 2 \\
$\# 13$ & 32 & 80 & 70 & 8 \\
\hline
\end{tabular}

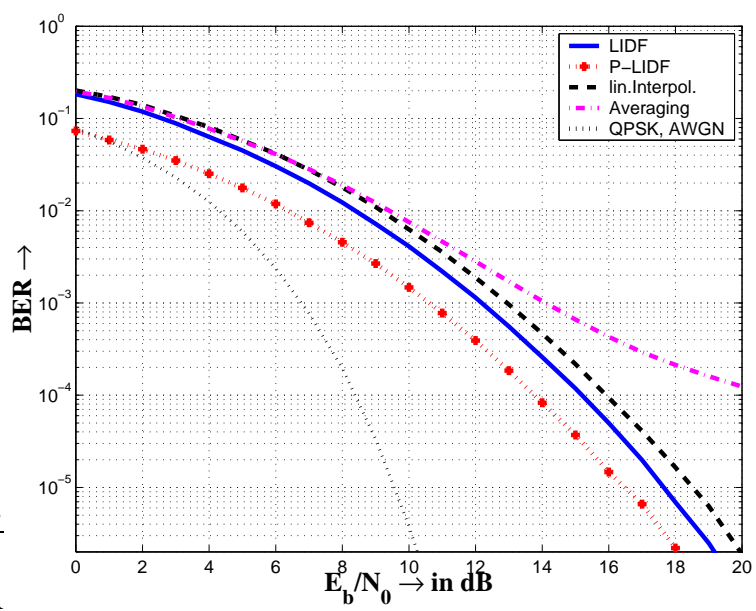

Figure 8: Vehicular-A slot format \# 8, v=120km/h

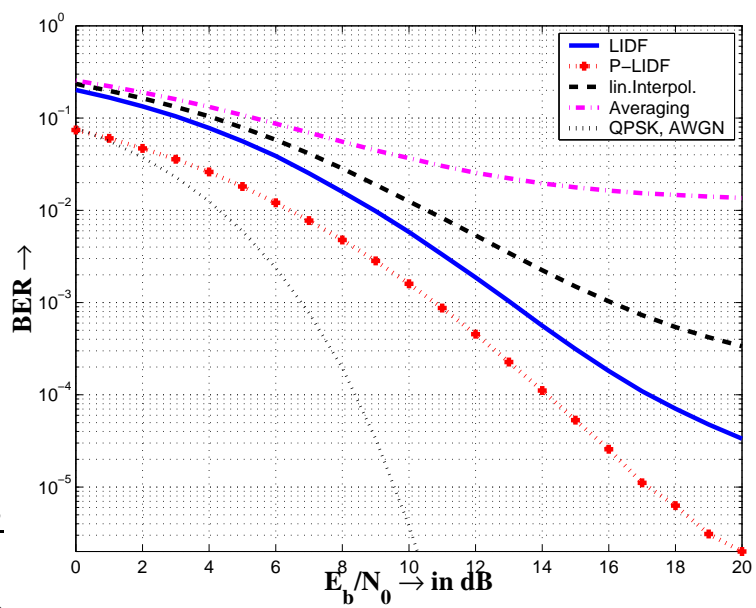

Figure 9: Vehicular-A slot format \# 8, v=300km/h

The Rake-receiver has six fingers but is only using taps for MRC that have at least $10 \%$ of the power of the most powerful tap, because very small taps are very hard to estimate due to crosstalk. Power control and channel coding are not in use. Next to the following simulation results the classical AWGN-curve is displayed. Besides the described LIDF scheme there is also a second one for reference that assumes a correct (perfect) predetection of data for channel estimation $\left(\hat{d}_{P o l, \nu}=d_{\nu}(i)\right)(\mathrm{P}-\mathrm{LIDF})$. Therefore the use of a FEC will result in a BER between the shown LIDF scheme without FEC and the one with perfect data decision.

In fig. 8 a Vehicular-A channel for a velocity of $120 \mathrm{~km} / \mathrm{h}$ and $S F=128$ is taken.

Here, LIDF provides the best results. These improvements are quite small compared to the classical CEs. Keep in mind that the number of data symbols in this format \#8 is also quite small (17 Symbols) compared to the number of pilot symbols (2). This results in a strong impact of false estimates on the LIDF scheme on the one hand and a very good linear interpolation/averaging on the other one. Because of this, LIDF degrades compared with perfect data detection significantly between $1 \mathrm{~dB}$ in a low noise environment and up to $3.5 \mathrm{~dB}$ in areas with strong noise. In this case using a FEC for data detection would probably improve the performance of this system very well.

Using this channel, averaging can not cope with the time variant channel, but its good noise reduction capabilities are sufficient in a noisy environment till $8 \mathrm{~dB}$.

Linear interpolation can cope with the time variant channel quite well. Its worse bit error rate up to an $E_{b} / N_{0}$ loss of $0.75 \mathrm{~dB}$ for very high $E_{b} / N_{0}$ compared to LIDF are due to its worse noise reduction.

In the case displayed in fig. 9 the velocity is raised to $300 \mathrm{~km} / \mathrm{h}$. At this high speed LIDF-CE outperforms the classical ones quite clearly. Even in a noisy environment the LIDF scheme has a gain of about one $\mathrm{dB}$ against the linear interpolation which raises with better $E_{b} / N_{0}$.

Averaging is totally overstrained with the time variance of the channel. Even its good noise reduction capabilities do not help, resulting in an error floor at about one percent.

Linear interpolation does compensate the change of channel coefficients much better. Nevertheless linear interpolation can not adapt completely to the time variant change, because this change can not be modelled as linear, which leads to an error-floor at about $2 \cdot 10^{-4}$.

Due to its linear approach LIDF-CE also can not cope with the time variance of the channel, but it tries to find an optimal line resulting in a better error floor at approximately $2 \cdot 10^{-5}$ and a much better performance compared to the classical schemes. Similar to the $120 \mathrm{~km} / \mathrm{h}$ case PLIDF also gains significantly. In fig. 10 and 11 the bit error rates for a Vehicular-A channel for $120 \mathrm{~km} / \mathrm{h}$ and $300 \mathrm{~km} / \mathrm{h}$ and $S F=32$ are displayed. The overall performance does naturally degrades with about $0.5-1 \mathrm{~dB}$ due to the lesser spreading factor. Linear interpolation and averaging look quite similar compared with the $S F=128$ case. The main difference in this case is that the P-LIDF scheme is not as good as before. Compared to non perfect LIDF its gain shrinks to about 0.5 to $1.5 \mathrm{~dB}$. Here, utilising a FEC for better data decision can not improve the performance that much compared to the $S F=128$ case. LIDF does have enough data symbols to deal with, therefore false data decisions do not effect the performance significantly.

In fig. 12 and 13 the BER is displayed in the presence of intracell multiuser interference. The BS transmits the signals for every user with the same power. The numbers of users are 16 and 32 and the spreading factor is 128 (slot 


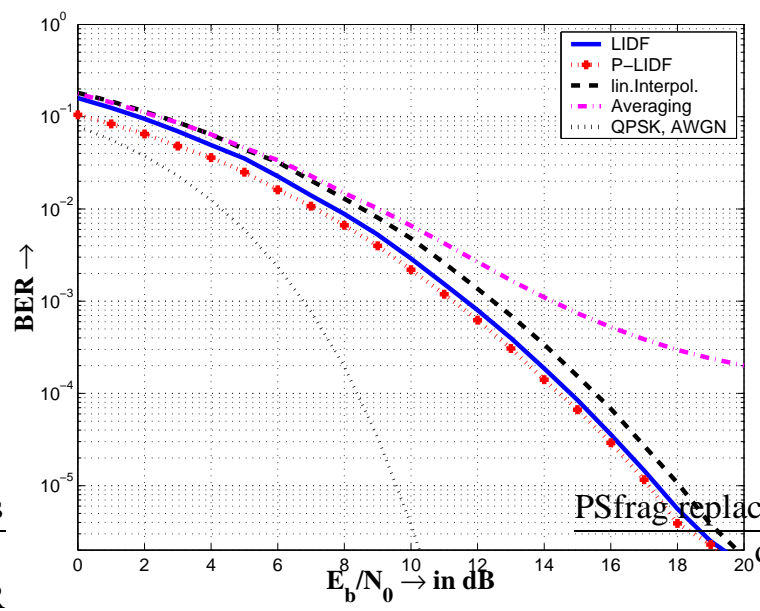

Figure 10: Vehicular-A slot format \# 13, v=120km/h

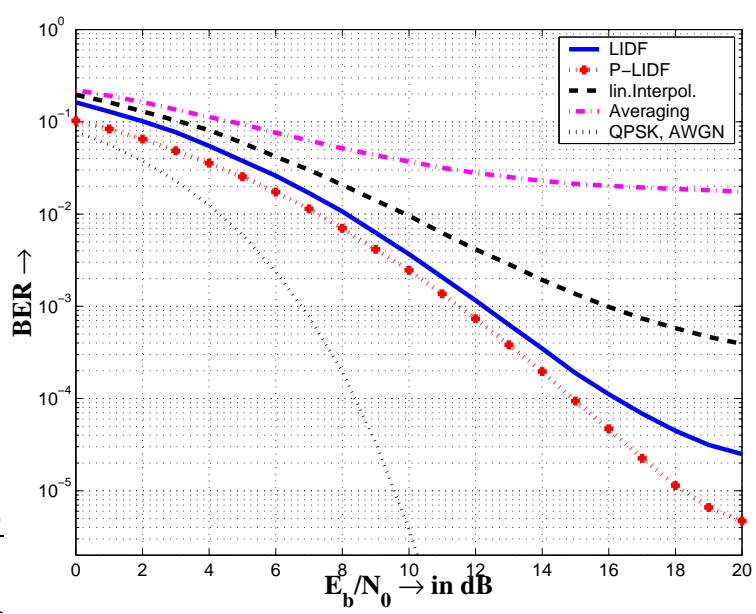

Figure 11: Vehicular-A slot format \# 13, v=300km/h

format \# 8). The performance degrades fast with raising number of users. Nevertheless the performance advantage of LIDF is mostly the same compared to the classical ones. The gain for $\mathrm{v}=120 \mathrm{~km} / \mathrm{h}$ is about $1.5 \mathrm{~dB}$ at an $E_{b} / N_{0}$ of 10 $\mathrm{dB}$ or even much higher in the $300 \mathrm{~km} / \mathrm{h}$ case. Since noise is gets more dominant with the number of users performance of lin. interpolation gets weaker compared to averaging.

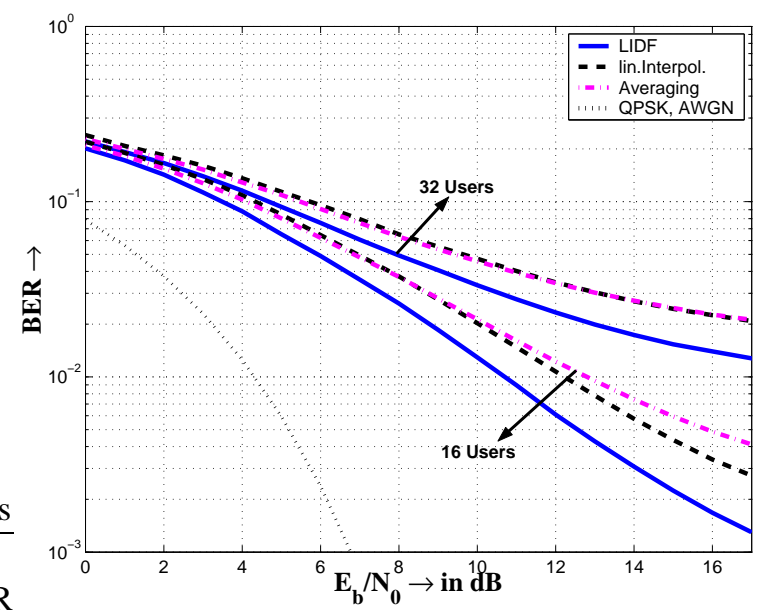

Figure 12: VHC-A, $S F=128, \mathrm{v}=120 \mathrm{~km} / \mathrm{h}, 16 \& 32$ Users

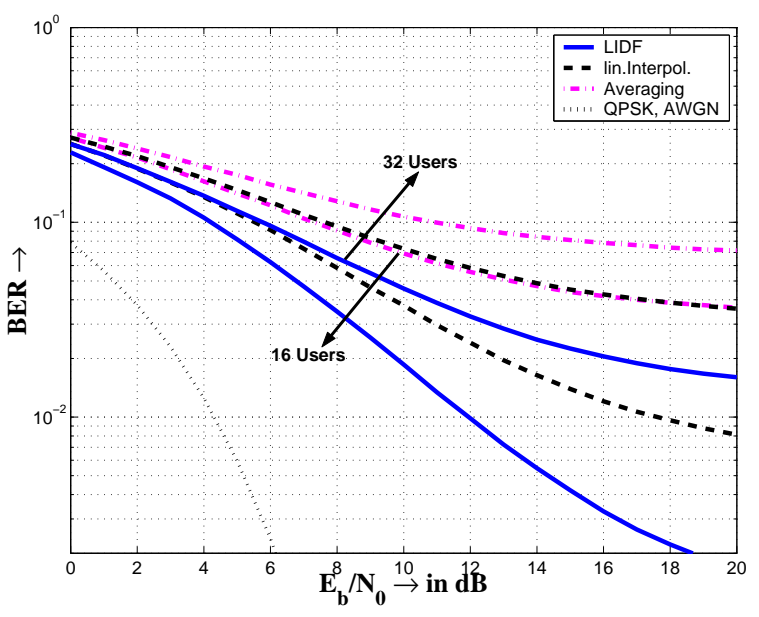

Figure 13: VHC-A, $S F=128, \mathrm{v}=300 \mathrm{~km} / \mathrm{h}, 16 \& 32$ Users

\section{Conclusions}

In general $\mathrm{CE}$ has to cope with noise and time variance of the channel. The here proposed LIDF-CE is capable to cope time variant channels and noise reduction. This paper shows the basic idea of the LIDF-CE and provides some clues of the utilizability, of such a scheme. It also considers multiuser conditions quite briefly.

For further studies there are still a few things which might be interesting.

First of all, linear regression can be used on Common Pilot Channel which can be quite beneficial. The problem of detection errors is gone and time variance within a slot can be compensated with the regression line while having better noise reduction capabilities. Additionally it may be an idea to consider the length of the regression line according to the velocity instead of taking exactly one frame. On the other hand for very high velocities higher order regression types may be examined.

As mentioned before the decision making process can be relocated after turbo- or convolutional decoding. This would lead to additional delay and higher computational cost but it would probably improve the performance in modes with higher spreading factors.

\section{REFERENCES}

[1] 3rd Generation Partnership Project. TS 25.211 Physical channels and mapping of transport channels onto physical channels (FDD), October 1999.

[2] G.T. Irvine and P.J. McLane. Symbol-Aided Plus DecisionDirected Reception for PSK/TCM Modulation on Shadowed Mobile Satellite Fading Channels. IEEE Journal on Selected Areas in Communications, 10(8):1289-1299, October 1992.

[3] Y. Honda and K. Jamal. Channel estimation based on TimeMultiplexed Pilot Symbols. Technical Report RCS96-70, IEICE, August 1996.

[4] L. Papula. Mathematik für Ingenieure und Naturwissenschaftler, volume 3. F. Vieweg, Braunschweig / Wiesbaden, 1994.

[5] European Telecommunications Standards Institute (ETSI). DTR/SMG-50402, Selection procedures for the choice of radio transmission technologies of the Universal Mobile Telecommunications System UMTS, 1997. Version 0.9.4. 